\title{
Broadband ZnO Single-Nanowire Light-Emitting Diode
}

\author{
Jiming Bao, Mariano A. Zimmler, and Federico Capasso* \\ Division of Engineering and Applied Sciences, Harvard University, \\ Cambridge, Massachusetts 02138
}

Xiaowei Wang and Z. F. Ren
Department of Physics, Boston College, Chestnut Hill, Massachusetts 02467

Received May 12, 2006; Revised Manuscript Received June 13, 2006

\begin{abstract}
We present a novel technique for reliable electrical injection into single semiconductor nanowires for light-emitting diodes and lasers. The method makes use of a high-resolution negative electron-beam resist and direct electron-beam patterning for the precise fabrication of a metallic top contact along the length of the nanowire, while a planar substrate is used as a bottom contact. It can be applied to any nanowire structure with an arbitrary cross section. We demonstrate this technique by constructing the first zinc oxide single-nanowire light-emitting diode. The device exhibits broad sub-bandgap emission at room temperature.
\end{abstract}

Semiconductor devices are found in a myriad of technological applications. These devices were made possible with the development of planar fabrication techniques, which allowed for the accurate control of their physical dimensions and, more importantly, their reproducibility. Semiconductor nanowire structures are now emerging as promising candidates for even further miniaturization, opening the door to interesting new functionalities. ${ }^{1,2}$ A powerful hybrid approach for engineering their optical properties by means of lithographic techniques has been reported recently. ${ }^{3}$ However, electrical injection into nanowires still remains a technical challenge. ${ }^{4}$ In this Letter, we address this issue by demonstrating a new method for achieving reliable electrical injection into a semiconductor nanowire. We show the potential of this technique by constructing the first zinc oxide $(\mathrm{ZnO})$ singlenanowire light-emitting diode (LED). Previous studies on $\mathrm{ZnO}$ nanowire LEDs were carried out on large numbers of nanowires simultaneously by defining a metallic contact on a thin film of nanowires. ${ }^{5,6}$

$\mathrm{ZnO}$ is a large band-gap $\left(E_{\mathrm{g}}=3.35 \mathrm{eV} \text { at } 300 \mathrm{~K}\right)^{7}$ semiconductor, with several desirable properties for nanowire laser diodes and LEDs, among many other applications. ${ }^{5,6,8-10}$ In particular, the high exciton binding energy $(60 \mathrm{meV})$, which is the result of a strong Coulomb interaction between electrons and holes, causes an enhancement of the radiative transition rate in the ultraviolet (UV) part of the spectrum. ${ }^{9,10}$ Furthermore, the electronic and optical properties of $\mathrm{ZnO}$ nanowires can be tailored by altering the growth conditions,

\footnotetext{
* Corresponding author. E-mail: capasso@deas.harvard.edu.
}

as well as by appropriate post-growth treatment. ${ }^{11}$ For example, as-made $\mathrm{ZnO}$ nanowires grown on sapphire substrates exhibit a prominent near band-edge UV peak, whereas nanowires grown on graphite flakes exhibit broad subbandgap luminescence. ${ }^{11}$ In addition, a single $\mathrm{ZnO}$ nanowire can form a resonant cavity with two naturally faceted hexagonal end faces acting as reflecting mirrors. ${ }^{9}$ The $\mathrm{ZnO}$ nanowires used in this study were grown by vaporizing and condensing a mixture of $\mathrm{ZnO}$ and graphite powder on carbon cloth. ${ }^{11,19,20}$ The resulting nanowires are n-type, similar to $\mathrm{ZnO}$ in bulk or thin films. ${ }^{15-17}$

Our technique (Figure 1) relies on the use of poly(methyl methacrylate) (PMMA) ${ }^{22}$ as a negative resist ${ }^{12,13}$ and electrically insulating layer. PMMA is commonly used as a positive resist for high-resolution electron-beam (e-beam) lithography. At lower doses, a process of polymer-chain scission dominates, and PMMA functions as a positive resist. In this case, the chain length of the polymer in the exposed area is reduced, making it more soluble in an appropriate solvent. At higher doses, however, a parallel process of polymerization becomes more dominant and PMMA can therefore be used as a negative resist. In this case, the polymer units are cross-linked together to form a hard insoluble material. The basic device geometry is depicted in Figure 1d, in which the n-type $\mathrm{ZnO}$ nanowire is placed between a heavily doped p-type silicon ( $\mathrm{p}-\mathrm{Si}$ ) wafer and a metallic thin film, serving as hole- and electron-injecting contacts, respectively. The cross-linked PMMA on the sides of the nanowire functions 
a.

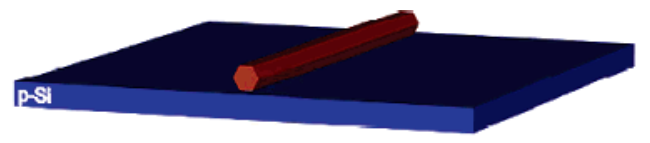

b.

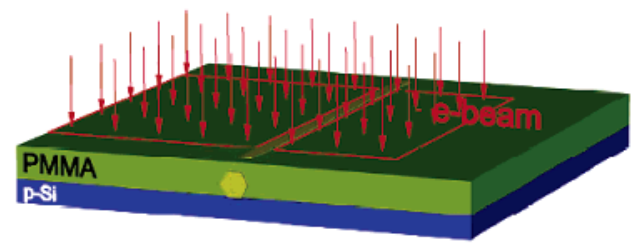

C.

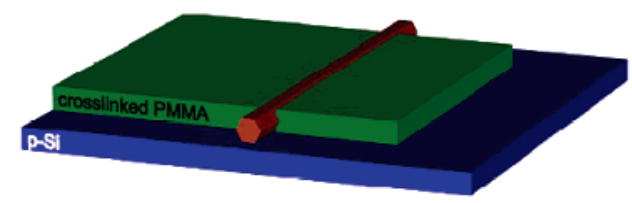

d.

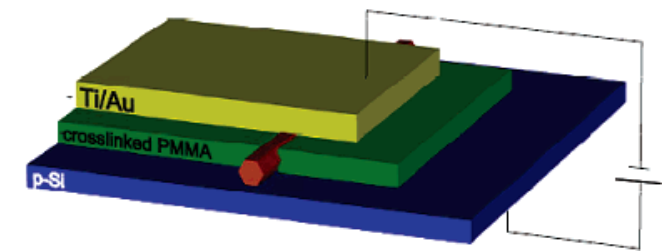

Figure 1. Schematic description of the procedure for fabricating a metallic contact on the top surface of a single nanowire. (a) $\mathrm{ZnO}$ nanowires are dispersed on a p-Si substrate. (b) A PMMA thin film $(\sim 120 \mathrm{~nm})$ is spin-coated on the substrate. The wire is located and imaged with the SEM of the focused ion beam (FIB) system. The pattern for e-beam exposure is defined directly on the SEM image (red outline), and subsequently written. The dose is $\sim 10$ $\mathrm{mC} / \mathrm{cm}^{2}$, which is 15 to 20 times higher than the dose for positive exposure. (c) The unexposed and partially exposed PMMA is removed by immersing the sample in acetone for $5 \mathrm{~min}$, then washed with IPA and dry cleaned with nitrogen air. Because PMMA shrinks when cross-linked, the thickness of the film reduces to $\sim 90$ nm. (d) Ti/Au is deposited with an electron-beam evaporator to form the top metallization. The geometry of the actual fabricated contact is shown in Figure 2.

as an insulating layer between the two contacts, thus allowing planarization and uniform electrical injection along the nanowire.

$\mathrm{ZnO}$ nanowires dissolved in ethanol are first randomly dispersed (Figure 1a) on a heavily doped p-Si substrate (resistivity is $\left.0.001 \mathrm{ohm}^{\circ} \mathrm{cm}\right)$. A thin film $(\sim 120 \mathrm{~nm})$ of PMMA is then spin-coated on the substrate. The key step involves patterning the PMMA in a manner such that it is cross-linked on both sides of the nanowire while leaving the top unexposed (Figure $1 \mathrm{~b}$ and $\mathrm{c}$ ). This is achieved with the electron beam of a dual-beam FEI focused ion beam (FIB) system, ${ }^{23}$ which allows direct imaging and writing. After e-beam writing, unexposed PMMA is stripped off with acetone. Figure $2 \mathrm{a}$ is a scanning electron microscope (SEM) image of a patterned $\mathrm{ZnO}$ nanowire, before metal deposition, which clearly shows the gap opened in the cross-linked PMMA revealing the top surface of the nanowire. Following an $\mathrm{O}_{2}$ plasma cleaning step to remove any organic residue on the exposed surface of the nanowire, Ti/Au is deposited
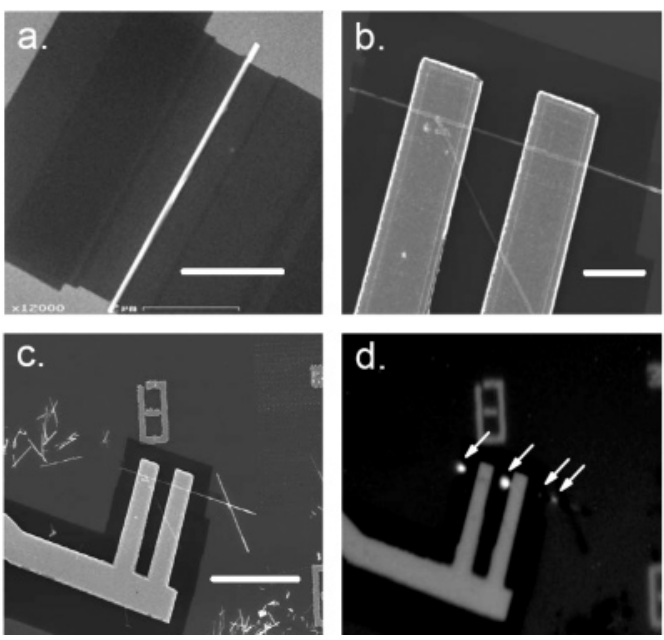

Figure 2. SEM and optical images of a single $\mathrm{ZnO}$ nanowire LED. (a) SEM image of a finished pattern on a $\mathrm{ZnO}$ nanowire before metal deposition (schematic shown in Figure 1c). Light gray, p-Si substrate; dark, cross-linked PMMA; white, $\mathrm{ZnO}$ nanowire. Scale bar is $2 \mu \mathrm{m}$. (b) SEM image of a typical finished device. The nanowire used to fabricate this device is different from the nanowire shown in a. The top Ti/Au metallic contact $(\sim 50 \mathrm{~nm} \mathrm{Ti}$ and $\sim 120$ $\mathrm{nm} \mathrm{Au}$ ) intercepts the wire with two prongs, leaving three open sections for light emission. The exposed top nanowire surface is visible. Note that the nanowire placed diagonally across the metallic pads is electrically insulated from the latter by a thin layer of crosslinked PMMA. The scale bar is $2 \mu \mathrm{m}$. (c) Zoomed-out SEM image of $b$. The less dark area is the p-Si substrate, and darkest one is the cross-linked PMMA. A stray $\mathrm{ZnO}$ nanowire intercepts the device on the right side. The scale bar is $10 \mu \mathrm{m}$. (d) Grayscale optical image of the device when a positive voltage $(7 \mathrm{~V})$ is applied to the substrate electrode with respect to the metallic contact. The light emission comes from four spots, indicated by the arrows.

with an electron-beam evaporator to form the top contact (Figure 1d). A total of over 10 devices were fabricated and tested. It was noticed that in the majority of the devices a rapid thermal annealing step $\left(\sim 300{ }^{\circ} \mathrm{C}\right.$ for $60 \mathrm{~s}$ in $\left.\mathrm{He}-\mathrm{H}_{2}\right)$ increased the forward current, leading to higher electroluminescence intensity.

The success of this technique relies on many factors, the most important of which is the precise alignment of the FIB electron beam. A small misalignment would cause the pattern to be shifted laterally, exposing the $\mathrm{p}$-Si electrode to the metallic contact and thus shorting the device. Other factors include the thickness of the PMMA film, e-beam dose, and acetone development time, all of which must be optimized to reduce the amount of PMMA that is cross-linked on top of the nanowire because of the proximity effect. With good e-beam alignment and an optimal recipe, we can routinely fabricate contacts on nanowires with diameters of $\sim 80 \mathrm{~nm}$. The smallest achievable separation between the two crosslinked PMMA sections ( $\sim 50 \mathrm{~nm}$ in our process) is primarily set by the electron-beam spot size and the proximity effect. The proximity effect is the result of electron scattering and causes the energy density deposited to spread as the beam passes through the resist. The beam spread is largest at the interface with the substrate, and thus this effect might be beneficial for larger wires because it would allow for adequate exposure of the PMMA blocked by the nanowire (assuming a hexagonal cross section, for example). 

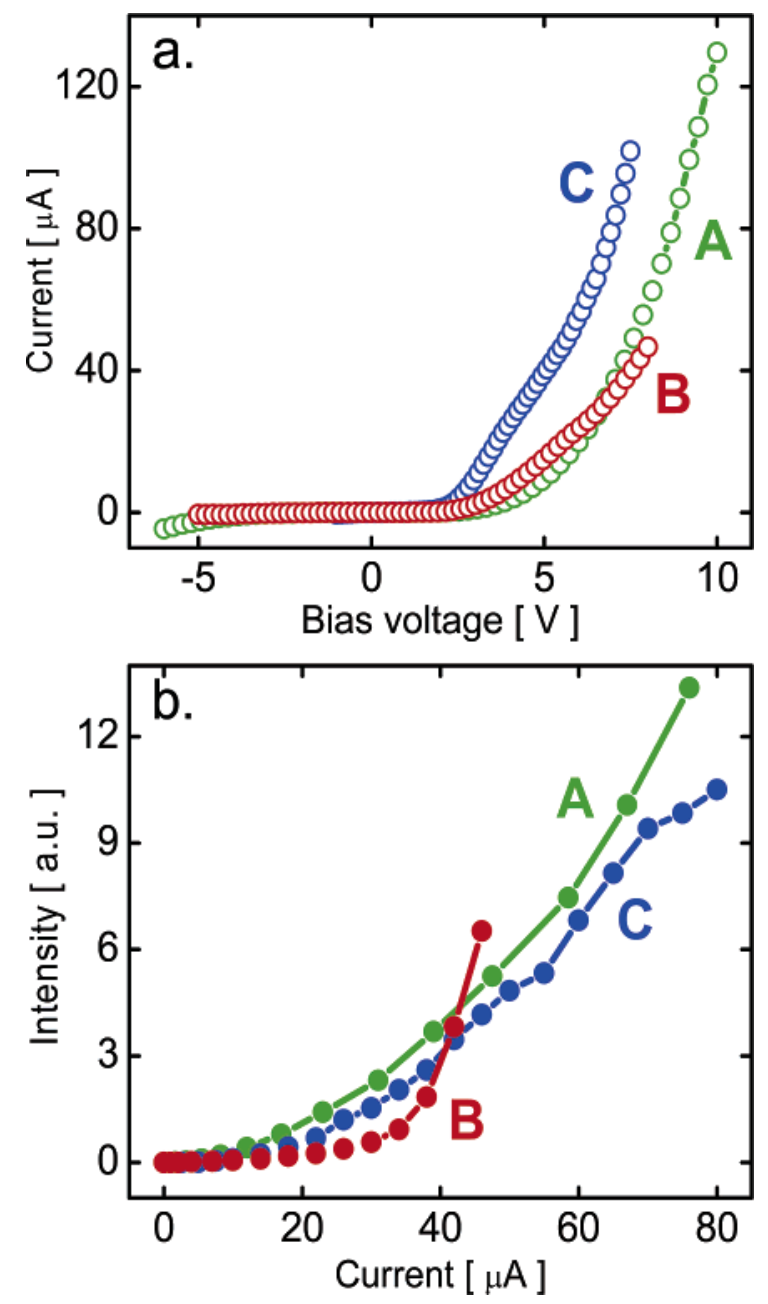

Figure 3. Current vs voltage and light intensity vs current characteristics of three finished devices. The solid lines are a guide to the eye. Device A corresponds to the device shown in Figure $2 \mathrm{~b}-\mathrm{d}$. The approximate contact areas are $\sim 0.27 \mu \mathrm{m}^{2}, \sim 0.16 \mu \mathrm{m}^{2}$, and $\sim 0.15 \mu \mathrm{m}^{2}$ for devices A, B, and C, respectively. (a) Current vs voltage characteristics at room temperature, in ambient air. Positive voltage corresponds to the voltage of the $\mathrm{p}$-Si substrate with respect to the top metallic contact. (b) Light intensity vs current for forward bias. Light was collected directly above the sample using a microscope objective with 50x magnification.

Figure $2 b$ and $c$ shows SEM images of a typical finished device (device A). An opening in the center of the top metallic contact was included to maximize the amount of light emission collected. Figure $2 \mathrm{~d}$ shows an optical image of the device when the substrate is biased positively with respect to the metallic contact (forward bias). The figure clearly shows that light emission originates from scattered spots along the nanowire (one in the middle opening, one at the left end of the nanowire, and two less intense spots on the right side, as indicated by the arrows) and not uniformly from the entire body. This observation emphasizes the waveguiding properties of these nanowires. We believe that the emission from the center opening and the two spots on the right side of the nanowire are due to local nonuniformities.

Figure 3a shows the current versus voltage $(I-V)$ characteristics for the same device shown in Figure $2 b-d$ (device A), as well as two other devices (device B and device C).

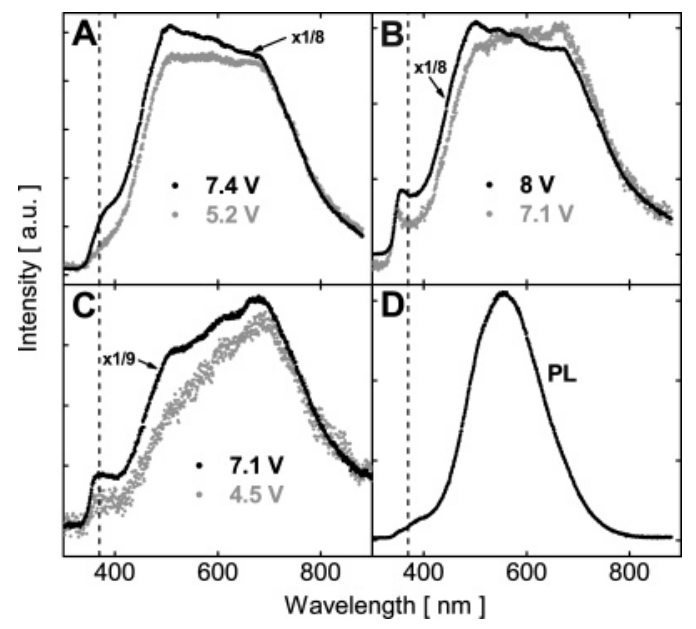

Figure 4. Photoluminescence and electroluminescence spectra of $\mathrm{ZnO}$ nanowires at room temperature, in ambient air. $\mathrm{A}-\mathrm{C}$ show the electroluminescence of three different devices (corresponding to devices A, B, and C, respectively, as shown in Figure 3) as a function of bias voltage. The higher bias spectra have been scaled by the indicated factor to display them on the same axis as the lower bias spectra. The dashed line indicates the wavelength of band-edge emission in bulk $\mathrm{ZnO}(370 \mathrm{~nm})$. (A) Electroluminescence spectrum of the single $\mathrm{ZnO}$ nanowire LED device (device $\mathrm{A}$ ) shown in Figure $2 \mathrm{~b}-\mathrm{d}$ at $5.2 \mathrm{~V}$ (gray) and $7.4 \mathrm{~V}$ (black). (B) Electroluminescence spectrum of a single $\mathrm{ZnO}$ nanowire LED (device $\mathrm{B}$ ) at $7.1 \mathrm{~V}$ (gray) and $8 \mathrm{~V}$ (black). (C) Electroluminescence spectrum of a single $\mathrm{ZnO}$ nanowire LED (device $\mathrm{C}$ ) at $4.5 \mathrm{~V}$ (gray) and 7.1 $\mathrm{V}$ (black). (D) Photoluminescence of a typical single $\mathrm{ZnO}$ nanowire.

The three devices show reasonable PN junction characteristics. Figure $3 \mathrm{~b}$ shows the measured light intensity versus current for the three devices. These two figures illustrate the reasonable reproducibility of the electrical and optical characteristics, within the nanowire variability. The observed differences could be due to the different diameters and lengths of the nanowires, in addition to the different contact areas. In particular, the measured luminescence will be strongly dependent on the outcoupling efficiency, which will be affected by nonuniformities along the nanowire length (Figure 2d). No significant variations in the current and emission intensity under a constant bias voltage were observed after several hours of continuous operation.

The single-nanowire electroluminescence (EL) spectrum was measured with a setup consisting of a microscope objective and a single-grating spectrometer equipped with a thermoelectrically cooled CCD camera. The photoluminescence (PL) spectrum was measured with the same setup and a $\mathrm{He}-\mathrm{Cd}$ laser $(325 \mathrm{~nm})$ as the excitation source. Figure 4 shows the EL for the devices shown in Figure 3 (devices $\mathrm{A}-\mathrm{C}$ ), at different voltages, as well as the PL of an isolated nanowire. The PL is centered at $556 \mathrm{~nm}$, and has a fullwidth at half-maximum (fwhm) of $\sim 170 \mathrm{~nm}$. The EL of all devices displays the same general features, extending from $350 \mathrm{~nm}$ to beyond $850 \mathrm{~nm}$. The weak luminescence centered at $\sim 380 \mathrm{~nm}$ has been attributed to excitonic recombination. $., 6,9,11,14,18$ The broad sub-bandgap emission in the PL and EL spectra is believed to be due to defects and surface states. ${ }^{5,6,11,18}$ The spectral characteristics are reproducible across different devices, and all three devices exhibit an 
enhancement of the short wavelength emission with increasing bias voltage.

This device structure can be identified as a $p-i-n$ junction. Oxygen molecules adsorb on the nanowire surface as negatively charged ions by capturing free electrons from the n-type $\mathrm{ZnO}$. With a nanowire radius comparable with the depletion layer thickness, the nanostructures can be assumed to be completely depleted. The dominant mechanism of electroluminescence is the recombination of electron and holes injected from the top metallic contact and the bottom substrate electrode, respectively. We should note, however, that under reverse bias we also have observed light emission, which we believe is due to impact ionization.

We should note that from the staggered band lineup at the $\mathrm{ZnO} / \mathrm{Si}$ interface that was inferred in recent work $^{21}$ this configuration is not ideal for an optimum device. The valence band discontinuity of $\sim 2.5 \mathrm{eV}$ strongly suppresses hole injection from the silicon substrate. ${ }^{21}$ This problem could be addressed by using alternate substrates with band gaps larger than that of $\mathrm{ZnO}$, such as p-type GaN.

In summary, we have demonstrated a new method for achieving reliable electrical injection into single semiconductor nanowires and we have used this technique to construct the first $\mathrm{ZnO}$ single-nanowire LED. The method can be applied to nanowires in a wide variety of materials.

Acknowledgment. We thank M. Loncar for helpful discussions, J. Xiang, W. Lu, Y. Huang, R. Agarwal, and M. S. Gudiksen for advice, and W. Yi, K. Russel, and V. Narayanamurti for the use of their facilities. We acknowledge financial support from DARPA under contract W911NF05-0-0286 and from the National Science Foundation Nanoscale Science and Engineering Center under contractPHY0017795. The support of the Center for Nanoscale Systems (CNS) at Harvard University is also gratefully acknowledged. HarvardCNS is a member of the National Nanotechnology Infrastructure Network (NNIN). The work performed at Boston College is supported by DOE grant DE-FG02-00ER45805.

\section{References}

(1) (a) Huang, Y.; Lieber, C. M. Pure Appl. Chem. 2004, 76, 2051. (b) Lieber, C. M. MRS Bull. 2003, 28, 486.
(2) (a) Samuelson, L.; Thelander, C.; Björk, M. T.; Borgström, M.; Deppert, K.; Dick, K. A.; Hansen, A. E.; Mårtensson, T.; Panev, N.; Persson, A. I.; Seifert, W.; Sköld, N.; Larsson, M. W.; Wallenberg, L. R. Physica E 2004, 25, 313. (b) Samuelson, L.; Björk, M. T.; Deppert, K.; Larsson, M.; Ohlsson, B. J.; Panev, N.; Persson, A. I.; Sköld, N.; Thelander, C.; Wallenberg, L. R. Physica E 2004, 21, 580. (c) Pettersson, H.; Trägårdh, J.; Persson, A. I.; Landin, L.; Hessman, D.; Samuelson, L. Nano Lett. 2006, 6, 229.

(3) Barrelet, C. J.; Bao, J.; Loncar, M.; Park, H.-G.; Capasso, F.; Lieber, C. M. Nano Lett. 2006, 6, 11.

(4) Duan, X.; Huang, Y.; Agarwal, R.; Lieber, C. M. Nature 2003, 421, 241.

(5) Konenkamp, R.; Word, R. C.; Schlegel, C. Appl. Phys. Lett. 2004, $85,6004$.

(6) Konenkamp, R.; Word, R. C.; Godinez, M. Nano Lett. 2005, 5, 2005.

(7) Sze, S. M. Physics of Semiconductor Devices, 2nd ed; WileyInterscience: New York, 1981.

(8) Kind, H.; Yan, H.; Mester, B.; Law, M.; Yang, P.; Adv. Mater. 2002, $14,158$.

(9) Huang, M. H.; Mao, S.; Feick, H.; Yan, H.; Wu, Y.; Kind, H.; Weber, E.; Russo, R.; Yang, P. Science 2001, 292, 1897.

(10) Bagnall, D. M.; Chen, Y. F.; Zhu, Z.; Yao, T.; Koyama, S.; Shen, M. Y.; Goto T. Appl. Phys. Lett. 1997, 70, 2230.

(11) Banerjee, D.; Lao, J. Y.; Wang, D. Z.; Huang, J. Y.; Steeves, D.; Kimball, B.; Ren, Z. F. Nanotechnology 2004, 15, 404.

(12) Zailer, I.; Frost, J. E. F.; Chabasseur-Molyneux, V.; Ford, C. J. B.; Pepper, M. Semicond. Sci. Technol. 1996, 11, 1235.

(13) Hoole, A. C. F.; Welland, M. E.; Broers, A. N. Semicond. Sci. Technol. 1997, 12, 1166.

(14) Zhang, B. P.; Binh, N. T.; Wakatsuki, K.; Segawa, Y.; Kashiwaba, Y.; Haga, K. Nanotechnology 2004, 15, S382.

(15) Vanheusden, K.; Warren, W. L.; Seager, C. H.; Tallant, D. R.; Voigt, J. A. J. Appl. Phys. 1996, 79, 7983.

(16) Collin, R. J.; Thomas, D. G. Phys. Rev. 1958, 112, 388.

(17) Oba, F.; Nishitani, S. R.; Isotani, S.; Adachi, H. J. Appl. Phys. 2001 90, 824.

(18) Shalish, I.; Temkin, H.; Narayanamurti, V. Phys. Rev. B 2004, 69, 245401.

(19) Banerjee, D.; Jo, S. H.; Ren, Z. F. Adv. Mater. 2004, 16, 2028.

(20) Jo, S. H.; Banerjee, D.; Ren, Z. F. Appl. Phys. Lett. 2004, 85, 1407.

(21) Mandalapu, L. J.; Xiu, F. X.; Yang, Z.; Zhao, D. T.; Liu, J. L. Appl. Phys. Lett. 2006, 88, 112108.

(22) 950-A4 PMMA was purchased from Microchem. The PMMA was diluted in anisole to obtain film thicknesses $\sim 120 \mathrm{~nm}$ by spin coating.

(23) Direct-write electron beam patterning was performed with an FEI DB235 FIB/SEM system. This system is equipped with a fieldemission electron beam and a $\mathrm{Ga}$ ion beam. High resolution was achieved with a $30 \mathrm{kV}$ accelerating voltage, $20 \mathrm{kx}$ SEM magnification, $30 \mu \mathrm{A}$ aperture size, and the minimum spot size. The beam current was measured to be $29 \mathrm{pA}$ with these settings. The exposure time is $\sim 3.6 \mathrm{~s}$ for a $1 \times 1 \mu \mathrm{m}^{2}$ pattern.

NL061080T 